\title{
Neuropathological Observations in a Patient with Carbamylphosphate-synthetase Deficiency and in Two Sibs
}

\author{
E. J. EBELS \\ From the Department of Pathology, University of Groningen, The Netherlands
}

\begin{abstract}
Ebels, E. J. (1972). Archives of Disease in Childhood, 47, 47. Neuropathological observations in a patient with carbamylphosphate-synthetase deficiency and in two sibs. Neuropathological findings are described in a child who suffered from carbamylphosphate-synthetase deficiency and died at the age of 7 months. The brain showed ulegyria of the cerebral and cerebellar cortex and hypomyelination of the centrum semiovale and the central part of the brainstem. Two sibs, who had died at the age of 4 weeks after a comparable illness, showed brain damage that seemed to represent an earlier stage of that observed in the first patient. These two children also showed bilateral symmetrical necrotizing lesions in certain brainstem areas (in one, kernicterus was observed macroscopically). The absence of further neuropathological observations in this disease makes it as yet impossible to reach any definite conclusion as to the aetiology of the brain lesions.
\end{abstract}

Recently in this journal, Hommes et al. (1969) published clinical and laboratory findings in a patient (Case 1) with deficiency of carbamylphosphate-synthetase (CPS), one of the enzymes of the Krebs-Henseleit or urea cycle. This patient died at the age of $7 \frac{1}{2}$ months. Two other sibs (Cases 2 and 3) had died at the age of 4 weeks after comparable though shorter illnesses. The remaining two children in the family are healthy and well. We report here the pathological findings on all three patients. As far as we know, neuropathological observations in this condition have not previously been published (apart from some very brief references by Freeman et al., 1970).

\section{Clinical Data}

Only the most relevant data are given here. For further details, especially the biochemical results, see Hommes et al. (1969).

Case 1. A girl born at term after an uneventful pregnancy, was admitted at age 20 days to hospital with feeding difficulties, which were the more alarming in view of the previous family history. She was extremely lethargic, with signs of minor convulsive activity and irregular eye movements. The tendon reflexes were very sluggish. Laboratory investigations showed slight metabolic alkalosis, low values for blood urea, and ammonia levels in the upper normal range. Serum and CSF arginine levels were significantly reduced. High blood glucose levels combined with low levels of pyruvate and lactate suggested a low glycolytic capacity. Liver biopsies, taken during life and after death, showed much reduced activity of CPS, with normal activity of the other urea cycle enzymes. A high ratio of ornithine transcarbamylase to CPS was also found in a piece of brain tissue removed immediately after death. After some temporary improvement (attributed to a change to a low protein diet) the patient gradually deteriorated. The EEG was normal at the time of admission, but later showed signs of increasing epileptic activity. Frank convulsions however did not occur during the illness, with the exception of terminal status epilepticus.

Cases 2 and 3. Little is known of the history of these 2 cases (a boy and a girl). Similar symptoms to those described for Case 1 occurred in the second week of life. Both infants died at the age of 4 weeks after closely comparable illnesses. Both showed signs of cerebral damage. The second infant had recurrent epileptic episodes during the last stage of the illness. Investigations that would have helped to establish the diagnosis were not carried out. 


\section{Morbid Anatomy}

Complete necropsies, including examination of the brain, were carried out on Cases 1 and 3 ; in the former, the spinal cord was also removed. Paraffin-embedded blocks of pieces of brain and other tissues from Case 2 were kindly put at our disposal by Dr. J. Sluiter, Amersfoort.

Case 1. Apart from the central nervous system, findings were limited to fatty changes in the liver and bronchopneumonia. Coronal slices of the brain revealed shrinkage and sclerosis of some cerebral gyri, especially at the border of the territories of the anterior and middle cerebral arteries in the frontal and parietal lobes on both sides. The white matter of the centrum semiovale was reduced in size and appeared to be of a firm consistency; some subcortical cavities were present, mainly in the regions of the sclerotic gyri. The corpus callosum was small.

At microscopical examination the shrunken gyri showed severe, often complete loss of neurones and increase in fibrillary astrocytes. This gliosis extended into the underlying white matter, reaching the lateral ventricles (Fig. 1). In some areas the atrophic cortex was separated from the white matter by cavities filled with macrophages There was no loss of nerve cells in the Ammon's horns. The thalamus on both sides showed extensive loss of neurones and fibrillary gliosis especially in the ventrolateral regions; some neurones were completely incrustated with calcium deposits. Extensive cell loss and gliosis were also seen in the dorsal areas of the putamen. The globus pallidus and subthalamic nucleus on both sides appeared normal. In the mesencephalon, the inferior colliculi, red nuclei, and substantiae nigrae were unaffected. In the pons and medulla, the central region showed a distinct loss of myelin associated with paucity of oligodendroglial cells: axons and neurones seemed to be present in normal number (Fig. 2). In the inferior olives some cell loss was present in the dorsomedial areas. Some cerebellar lobules were shrunken, with complete absence of Purkinje cells and fibrillary gliosis in the molecular layer. The dentate nuclei showed loss of neurones. In myelin-stained preparations the centrum semiovale appeared to be poorly myelinated. This was also evident in the anterior commissure and the corpus callosum. The spinal cord did not reveal any abnormalities. Alzheimer type I or II astrocytic changes were not present.



Fig. 1.- (Holzer stain $\times 2 \cdot 75)$ Case 1: severe gliosis is present in the shrunken gyri (arrows) and in the underlying white matter. 




FIG. 2.- $($ PTAH stain $\times 5)$ Case 1: hypomyelination of the central part of the pons.

Case 3. The liver showed fatty changes. There was some bronchopneumonia. In the kidneys sclerotic glomeruli were present in a fairly large number. In coronal slices a distinct yellow discoloration was observed in the thalamus and-less conspicuously-in the subthalamic nuclei, substantiae nigrae, and around the aqueduct. Microscopical examination showed widespread degeneration of neurones in the cerebral and cerebellar cortices, in the thalamus, basal ganglia, and brainstem. This degeneration appeared to be recent and of the ischaemic/anoxic type. Oligodendroglial cells often showed similar signs of degeneration. The neuropil appeared to be vacuolated and spongy, and some reaction of glial cells (astrocytes, microglia) was observed. In some regions a much more severe type of degeneration was present with complete loss of neurones, severe spongiosis, and glial reaction, and some increase in the number of capillaries (Fig. 3). This type of lesion was seen in the lateral thalamus, red nuclei, inferior colliculi, and the region including the superior olivary nuclei. These lesions were strikingly symmetrical. In some areas, especially the arterial boundary zones, the cerebral cortex showed complete loss of neurones and replacement of the parenchyma by astrocytes and macrophages (Fig. 4). Axons in general seemed to be well preserved with the exception of the severely affected regions in the brainstem, in which they showed fragmentation. In this case, too, Alzheimer type of glial reaction was absent.
Case 2. The necropsy data in this patient were limited. As far as we could assess they were essentially similar to those seen in Case 3 , both in the central nervous system and in the other organs. Symmetrical areas of degeneration in the brainstem, as observed in Case 3, were present in this patient, too.

\section{Discussion}

The comparable clinical histories of the 3 sibs make it reasonable to assume that they all suffered from the same inborn error of metabolism that was demonstrated in the most recent case (Case 1). The longer duration of the illness in this child may have been due to a less severe metabolic derangement and/or better guided clinical treatment which included a low protein diet.

In all 3 infants severe brain damage was present. In Case 1 the changes fit the concept of ulegyria (or Malamud's 'primary subcortical pathology' -Malamud et al., 1964). The only other case of ulegyria associated with an inborn error of metabolism known to us is that of Coulson and Bray (1969) of ulegyria combined with phenylketonuria. But here we may safely assume a fortuitous combination, as many cases of phenylketonuria are known without ulegyria. The very scarcity of neuro- 


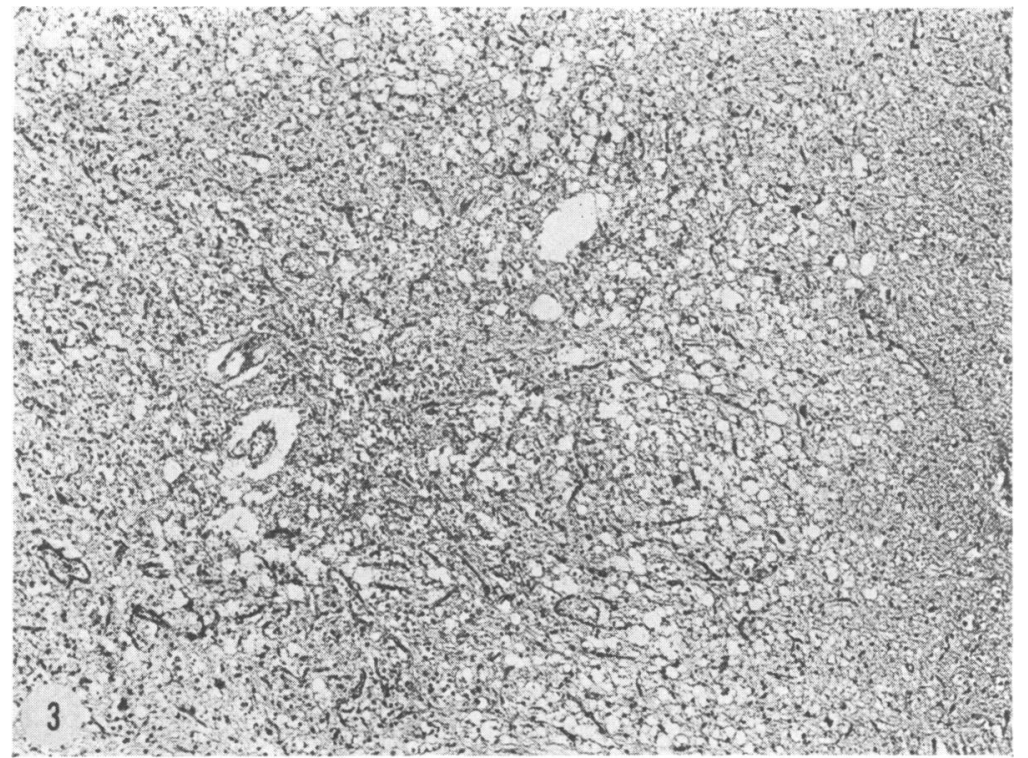

Fig. 3.-(H. and E. stain $\times 55)$ Case 3: degeneration of the inferior colliculus with loss of neurones, vacuolation and reaction of glial cells and capillaries.

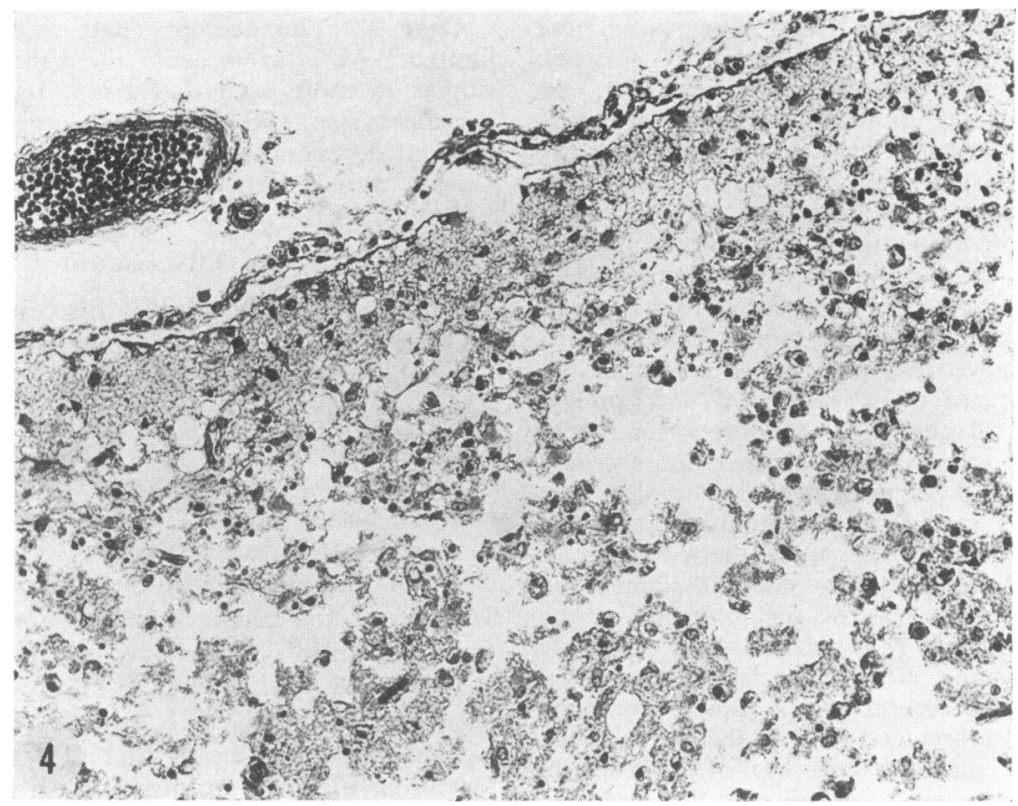

FIG. 4.-(H. and E. stain $\times 145)$ Case 3: severely affected area in the cerebral cortex with complete loss of neurones and disintegration; only subpial astrocytes and macrophages are present. 
pathological observations in CPS deficiency, however, makes it impossible to decide whether or not this holds for this metabolic error, too. The distribution of the lesions in the brain of Case 3, who died at a much younger age, corresponds closely to that observed in the ulegyric brain, though the damage here is of a much more acute or subacute type. In this case, too, the lesions in the cerebral cortex are especially severe in the arterial boundary zones. In both younger patients (Cases 2 and 3) severe lesions were present in addition in the brainstem showing the pattern of Poser and van Bogaert's 'polioencéphalopathie symétrique nécrotisante' (Poser and van Bogaert, 1960). It is noteworthy that this latter type of brain damage is observed in a variety of conditions often of a metabolic type, such as Leigh's disease. The striking yellow discoloration of brainstem areas observed in Case 3 hasin the absence of any reason to assume the presence of hyperbilirubinaemia-to be interpreted as secondary bilirubin-incrustation of severely damaged neurones. The observation by Freeman et al. (1970) of unexplained high levels of bilirubin in a patient with CPS deficiency and a sib suffering from an identical illness is of interest in this respect, however.

On comparing our cases, it seems that the pathological changes in the brain of Case 1 who died at $7 \frac{1}{2}$ months represented an inactive, residual stage, whereas those observed in Case 3 and Case 2 who died at 4 weeks represented an earlier, acute or subacute, stage of the same pathogenetic process. This holds for the alterations in the cerebral and cerebellar cortex. But also, the hypomyelination of the centrum semiovale and the brainstem in Case 1 may very well have been the result of direct damage to the oligodendroglial cells or their precursors as observed in Cases 2 and 3.

Freeman et al. (1970) very briefly state that in their patient with CPS deficiency and the sib who died after a similar illness, no significant abnormalities were found in the brain. Bruton, Corsellis, and Russell (1970) published neuropathological data of 2 patients with ornithine transcarbamylase deficiency that are of obvious interest, as the metabolic derangement resulting from this deficiency can be assumed to be very similar to that in CPS deficiency. In both patients they found marked
Alzheimer type II changes in astrocytes, such as are observed in many conditions in which blood ammonia levels are raised. We did not observe these in our patients. The explanation may be that in our patients the blood ammonia levels were only moderately increased. Cavanagh and Kyu (1971) demonstrated in rats with experimental portocaval shunts that the degree of Alzheimer type II astrocytic changes shows a direct positive correlation with the blood ammonia level. In their Case 1, however, Bruton et al. (1970) in addition, described other, very severe, lesions which correspond closely to those observed in Case 1.

\section{Conclusion}

In all 3 sibs, severe brain damage was present. In one of two cases with a closely related inborn error, Bruton et al. (1970) showed comparable brain damage.

At present, however, it is impossible to decide what relation, if any, exists between the metabolic error and the neuropathological findings described. For the time being, the value of publishing necropsy reports lies in the accumulation of data that will ultimately enable us to distinguish between essential and concomitant changes. Only then will there be justification for speculating about the aetiological mechanisms involved.

\section{REFERENCES}

Bruton, C. J., Corsellis, J. A. N., and Russell, A. (1970). Hereditary hyperammonaemia. Brain, 93, 423.

Cavanagh, J. B., and Kyu, M. H. (1971). Type II Alzheimer change experimentally produced in astrocytes in the rat. Fournal of the Neurological Sciences, 12, 63.

Coulson, W. F., and Bray, B. F. (1969). An association of phenylketonuria with ulegyria. Diseases of the Nervous System, 30, 129.

Freeman, J. M., Nicholson, J. F., Schimke, R. T., Rowland, L. P., and Carter, S. (1970). Congenital hyperammonemia: association with hyperglycinemia and decreased levels of carbamyl phosphate synthetase. Archives of Neurology, 23, 430.

Hommes, F. A., de Groot, C. J., Wilmink, C. W., and Jonxis, J. H. P. (1969). Carbamylphosphate synthetase deficiency in an infant with severe cerebral damage. Archives of Disease in Childhood, 44, 688.

Malamud, N., Itabashi, H. H., Castor, J., and Messinger, H. B. (1964). An etiologic and diagnostic study of cerebral palsy. Fournal of Pediatrics, 65, 270.

Poser, C., and van Bogaert, L. (1960). Leuco- et polio-encéphalopathies symétriques nécrosantes. Revue Neurologique, 103, 3.

Correspondence to Dr. E. J. Ebels, Division of Neuropathology, Department of Pathology, University of Groningen, The Netherlands. 